\title{
Comparative, randomized, pharmacoeconomic evaluation of sequential antibiotic therapy at the Centre Hospitalier de I'Université Laval, Sainte Foy, Quebec: A unique approach
}

\author{
MG BERGERON MD, AY MARTEL MD, L BERGERON CPH, M VALOIS MSC
}

$\mathrm{T}$ HE CONCEPT OF INTRAVENOUS TO ORAL ANTIMICROBIAL stepdown therapy has been evolving over the past few years. Despite many publications describing a positive economic impact of this approach, there has been no evaluation of the overall economic consequences of this strategy on health care, other than drug acquisition costs. Furthermore, the vast majority (if not all) of these programs require the intervention of an infectious diseases physician and/or a pharmacist who evaluates the antimicrobial therapy and intervene directly with the physician responsible for the patient. This represents not only added expenses that may lessen the cost-avoidance of the stepdown program but also a major impediment on the applicability of this strategy in community hospitals or other health care organizations where there are no infectious diseases specialists or pharmacists with a sufficient degree of expertise in antimicrobial therapy. We prefer the use of sequential antibiotic therapy (SAT) instead of 'stepdown' as we believe it represents what is really happening.

\section{STUDY OBJECTIVE}

The objective of our study is to determine whether simple written suggestions proposing oral alternatives

Laboratoire et Service d'Infectiologie, Centre Hospitalier de l'Université Laval, Sainte-Foy, Québec

Correspondence: Dr Michel G Bergeron, Laboratoire et Service d'Infectiologie, Centre Hospitalier de l'Université Laval, 2705 boulevard Laurier, Sainte-Foy, Québec G1V 4G2, telephone 418-654-2705, Fax 418-654-2715 for intravenous antibiotics, chosen from a pre-established list of oral equivalents, is effective in inciting physicians to switch more rapidly to the oral route. We also seek to determine if this strategy results in cost reduction, using the concept of disease costing (eg, all the expenses related to the treatment of an illness, including the cost of medical consultation, imaging, hospital maintenance, etc).

\section{STUDY DESIGN}

Inclusion and exclusion criteria: Patients included are adult patients (older than 18 years) for whom parenteral antibiotics are prescribed. Reasons for exclusion are the following: cystic fibrosis, cystitis or pyelonephritis, ophthalmic infection, meningitis or any other central nervous system infection, AIDS, or patient under care of one of the investigators (MGB, AYM).

Pre-study control group: In order to assess the influence of our interventions on prescription habits (are physicians using more intravenous to oral stepdown after the implementation of our study?), we constituted a control group during the three months preceding the beginning of the study. We did not make any active intervention for these patients and only collected data (see below).

Experimental phase: Patients eligible for inclusion in the study are stratified by infection site, and randomized within each stratum into the observation or intervention group. We follow the same passive procedure as the prestudy control group for the observation group 
(eg, data collection only). For patients included in the intervention group, a standardized form is inserted on top of each individual medical record. This form proposes at least two oral alternatives and their respective daily cost as well as the daily cost of the intravenous antibiotics administered to the patient. These suggestions are made using a standardized, pre-established list of oral equivalents. Data concerning the evolution of the infection (white blood cell count, temperature, APACHE score) and the costs involved (antibiotics, laboratory tests, length of stay, complications) for the entire patient stay in the hospital are collected for each patient in both experimental groups and for the prestudy control group.

\section{PRELIMINARY RESULTS}

More than 300 patients have been included in our study since September 1993. We present here preliminary results from early analysis of the first 64 patients randomized into the intervention group. These patients were suffering mainly from intra-abdominal and pulmonary infections.

Physicians tend to follow our suggestions more often when single drug therapy is used (70\% [19 of 27] followed), whereas the proportion of suggestions followed was only $51 \%$ (19 of 37) when combination therapy was used. When the intervention is followed and single drug therapy is used, beta-lactams are used preferentially both for intravenous and oral routes, with the exception of third generation cephalosporins for which oral quinolones are most commonly preferred.

When the intervention is not strictly followed, the most common reasons are early stepdown therapy, discharge of the patient without antibiotics, discontinuation of antibiotics, and a clinical condition necessitating intravenous therapy in the opinion of the physician. In fact, we have noticed that many of the above changes in management occurred following our intervention. The sample size is too small yet to know whether or not it is related to our intervention.

\section{CONCLUSION}

Among the characteristics that distinguish our approach from conventional stepdown strategy, the following are the most distinctive:

- the presence of a prestudy control group is unique

- patient randomization into an intervention and observation (or experimental and control) group has not been done before. This approach should permit us to assess adequately the real impact of our intervention on prescription trends.

It is obviously too early in the data analysis process to be able to draw conclusions, but we believe that our experimental approach will allow a more thorough pharmacoeconomic evaluation of this complex issue. 


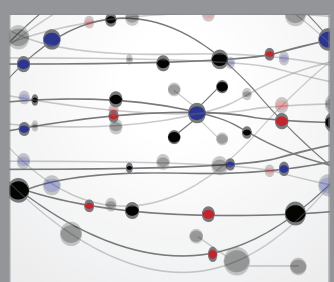

The Scientific World Journal
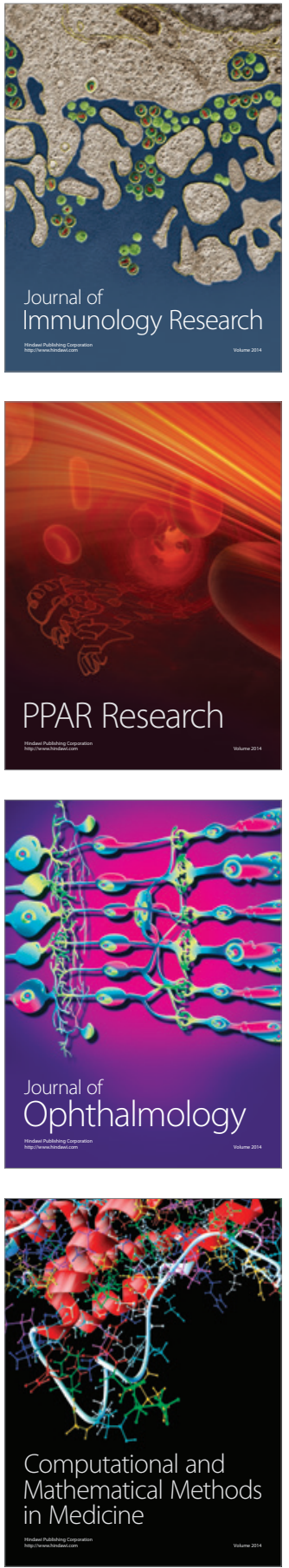

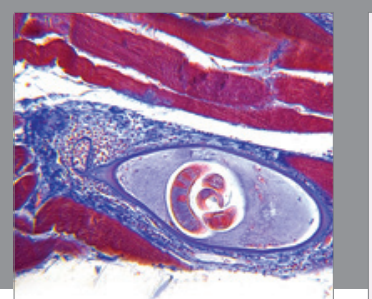

Gastroenterology Research and Practice

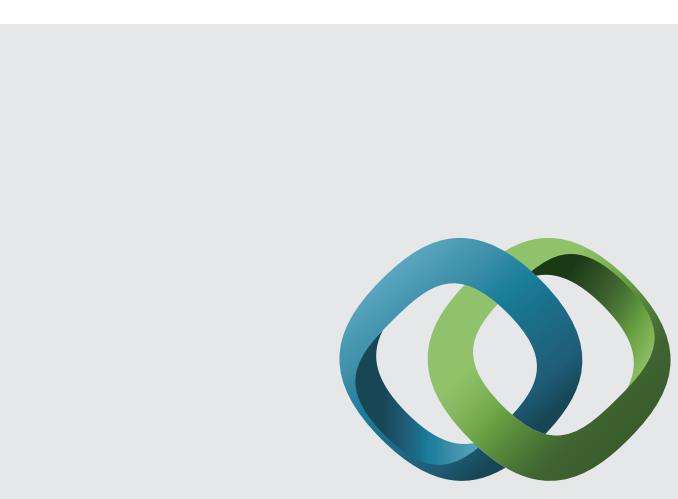

\section{Hindawi}

Submit your manuscripts at

http://www.hindawi.com
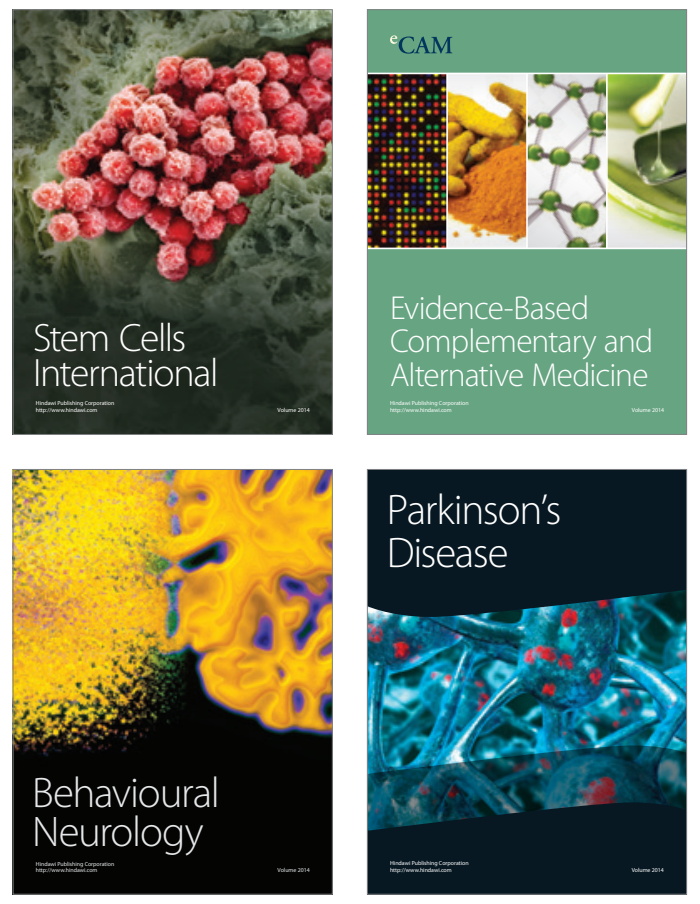
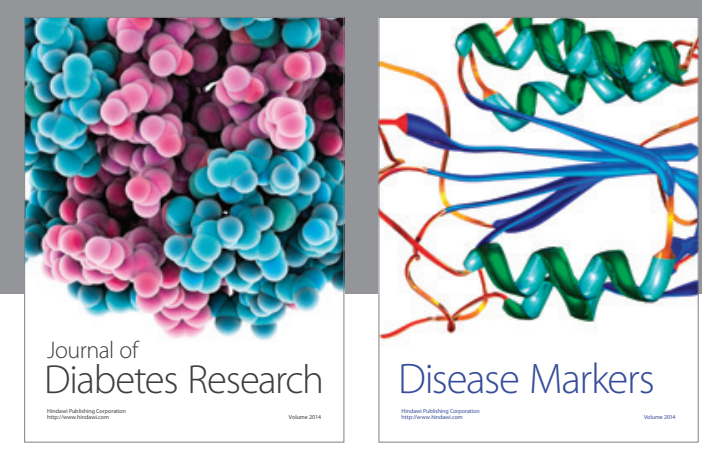

Disease Markers
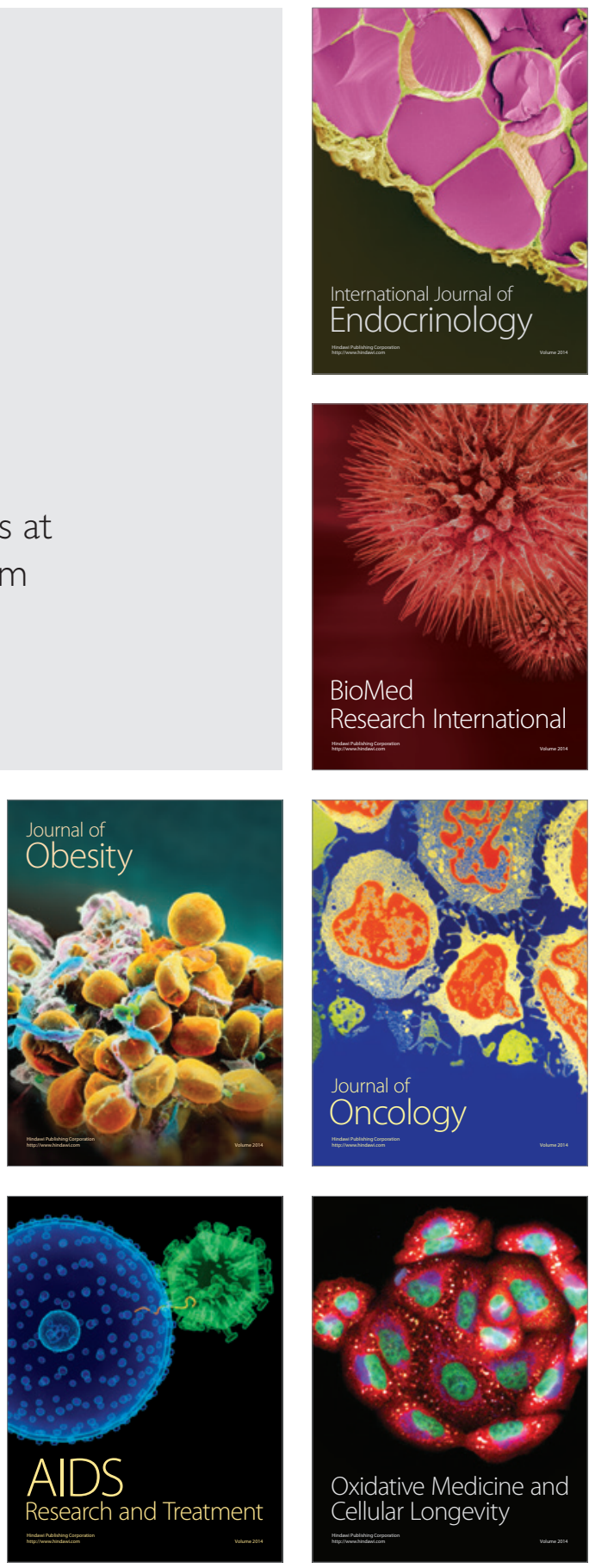